\title{
Jet Physics and the Underlying Event at the Tevatron
}

\author{
Rick Field ${ }^{1}$ \\ (for the CDF \& D0 Collaborations)
}

Department of Physics, University of Florida, Gainesville, Florida, 32611, USA

\begin{abstract}
Tevatron Run 2 results on the inclusive jet cross section (MidPoint and $\mathrm{K}_{\mathrm{T}}$ algorithm) and the b-jet and $b \bar{b}$-jet cross section (MidPoint algorithm) are presented and compared with theory. The CDF b-jet $\bar{b}$-jet $\Delta \phi$ distribution is compared with theory and with the D0 jet\#1-jet\#2 $\Delta \phi$ distribution. The understanding and modeling of the "underlying event" in Run 2 at the Tevatron is reviewed and new CDF results are presented.
\end{abstract}

Keywords: QCD, Jets, Hadron Collider.

PACS: $12.38 .-$ t, $12.38 . \mathrm{Bx}, 12.38 . \mathrm{Qk}$.

The study of proton-antiproton collisions in Run 2 at the Tevatron is teaching us a lot about how QCD works. Comparing data with theory will lead to improved QCD Monte-Carlo models and to more precise parton distribution functions. In Run 2 at the Tevatron we are studying the inclusive jet cross section using both the MidPoint cone algorithm and the $\mathrm{K}_{\mathrm{T}}$ algorithm [1]. We are studying heavy flavor jets (i.e. b-jets) and jets produced in association with photons, $\mathrm{W}$ bosons, and $\mathrm{Z}$ bosons. We are studying jet-jet correlations, jet fragmentation (jet shapes, momentum distributions, two-particle correlations), and we are making good progress in understanding and modeling the "underlying event" in hard scattering processes. Here I will only be able to show a little bit of what we have learned.

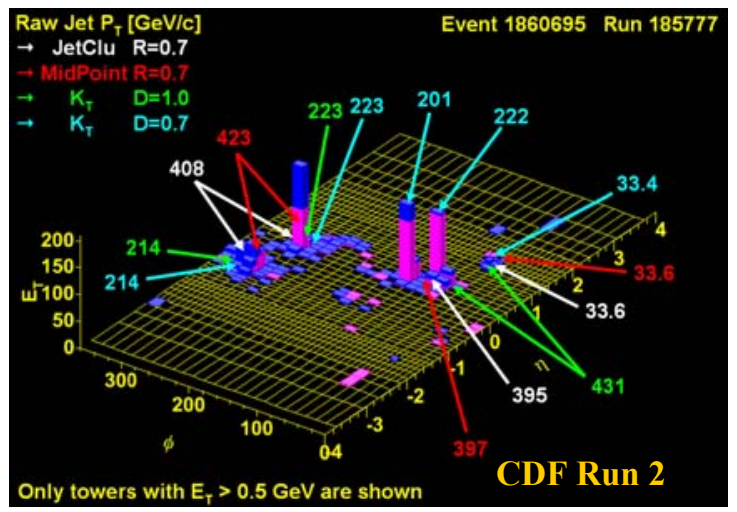

FIGURE 1. Shows the transverse energy of calorimeter towers with $\mathrm{E}_{\mathrm{T}}>0.5 \mathrm{GeV}$ for an event in the CDF detector. The MidPoint algorithm combines the two clusters into one "jet" with $\mathrm{p}_{\mathrm{T}}=423 \mathrm{GeV} / \mathrm{c}$ while the $\mathrm{K}_{\mathrm{T}}$ algorithm $(\mathrm{D}=0.7)$ finds two "jets" with $\mathrm{p}_{\mathrm{T}}=223 \mathrm{GeV} / \mathrm{c}$ and $214 \mathrm{GeV} / \mathrm{c}$.

${ }^{1}$ To appear in the proceedings of the XXXV International Symposium on Multiparticle Dynamics, Kromeriz, Czech Republic. 
Experimentally we measure "jets" at the detector (i.e. calorimeter) level by observing the energy in each calorimeter cell as illustrated in Fig. 1. Of course the "jet" cross section depends on ones choice of jet algorithm. Each jet algorithm is a different observable and comparing the results of different jet algorithm teaches us about QCD. Of course, what is measured in the calorimeter must be corrected for detector efficiency which is done by comparing the QCD Monte-Carlo models at the particle (i.e. generator level) with the result after detector simulation. I believe that experimenters should publish what they measure (i.e. observables at the particle level with the "underlying event"). However, to compare with NLO parton level calculations one must go one step further. The NLO parton level does not have fragmentation or an "underlying event" (i.e. beam-beam remnants, initial and finalstate parton showers, multiple-parton interactions, resonance decays, etc.). There are three approaches for comparing data corrected to the particle level (i.e. hadron level) with parton level calculations. The first approach is to neglect the difference and to compare the hadron level data directly with the parton level calculation. Fig. 2 shows the inclusive jet cross section using the MidPoint algorithm $\left(R=0.7, f_{\text {merge }}=0.5\right)$ for two rapidity bins as measured by D0. D0 compares the experimentally measured hadron level prediction directly with the NLO parton level theory curves and assumes that the parton level to hadron level corrections are small for jets above $50 \mathrm{GeV}$. The agreement between the parton-level theory prediction and the measured hadron-level is quite good over 10 decades!
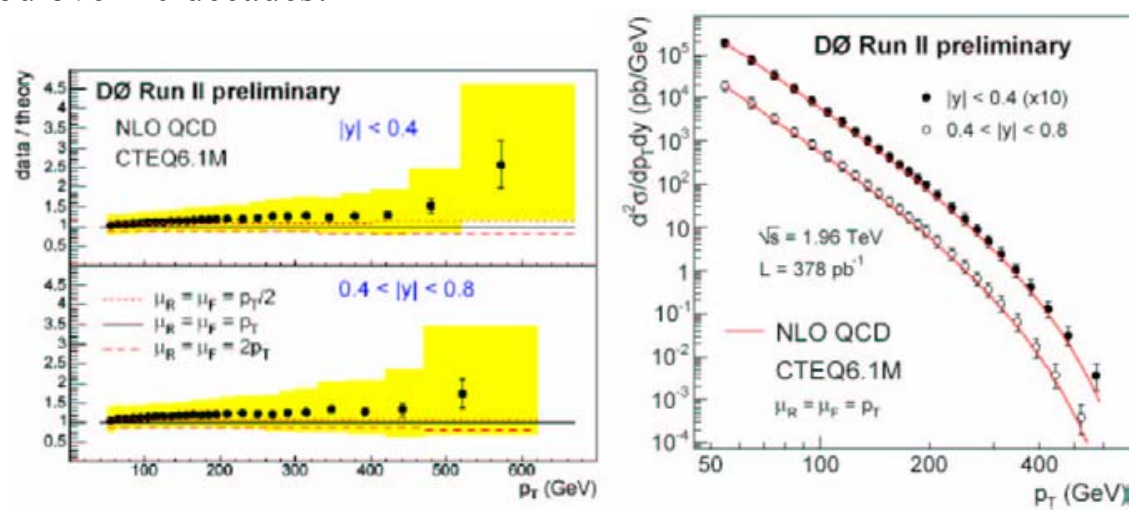

FIGURE 2. The D0 Run 2 inclusive jet cross section using the MidPoint algorithm $\left(\mathrm{R}=0.7, \mathrm{f}_{\text {merge }}=0.50\right)$ compared with parton-level NLO QCD. The hadron-level data are compared directly with the parton-level NLO QCD.

Another approach for comparing what is measured at the particle level in the detector with the NLO parton level theory is to use the QCD Monte-Carlo models and try to extrapolate the data to the parton level. This requires removing the "underlying event" and correcting for fragmentation effects. Fig. 3 shows the inclusive jet cross section using the MidPoint algorithm $\left(\mathrm{R}=0.7, \mathrm{f}_{\text {merge }}=0.75\right)$ in the central region as measured by CDF compared with the parton level NLO QCD prediction, where the data have been extrapolated (i.e. corrected) to the parton level. Fig. 3 shows that the hadron level to parton level correction factors are significant for $\mathrm{P}_{\mathrm{T}}(\mathrm{jet})<300 \mathrm{GeV} / \mathrm{c}$ (they come mostly from the "underlying event"). The agreement between the theory and data is very good. 

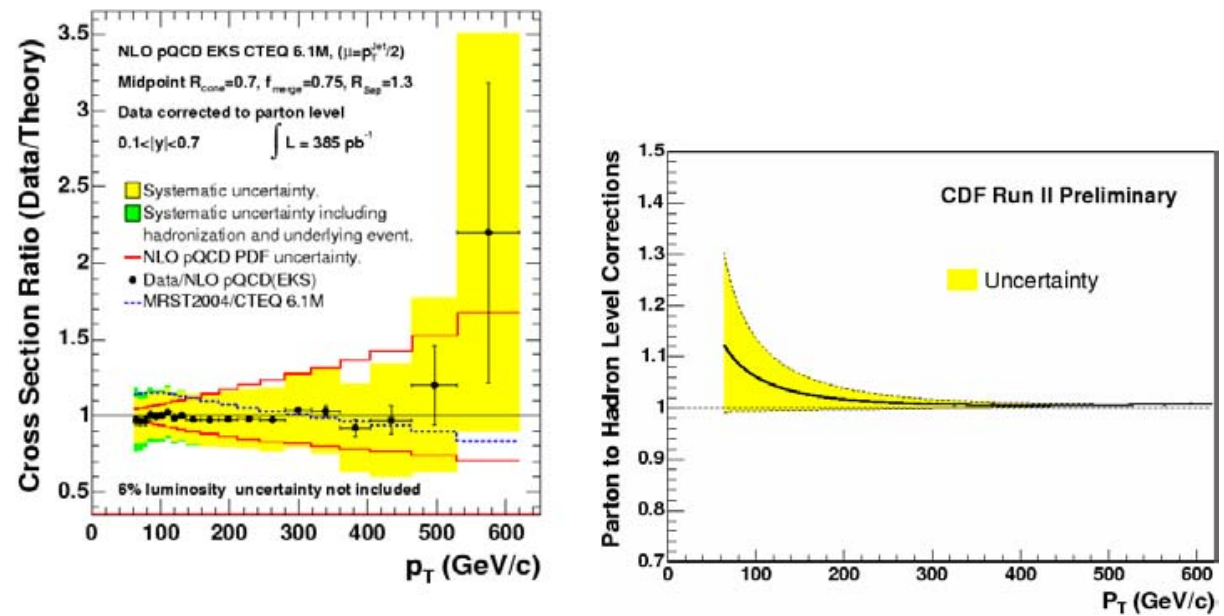

FIGURE 3. The CDF Run 2 inclusive jet cross section using the MidPoint algorithm $\left(\mathrm{R}=0.7, \mathrm{f}_{\text {merge }}=0.75\right)$ compared with parton-level NLO QCD (left). The data have been extrapolated (i.e. corrected) to the parton level using the parton to hadron correction factor (right). The hadron-level data are multiplied by the reciprocal of this factor.
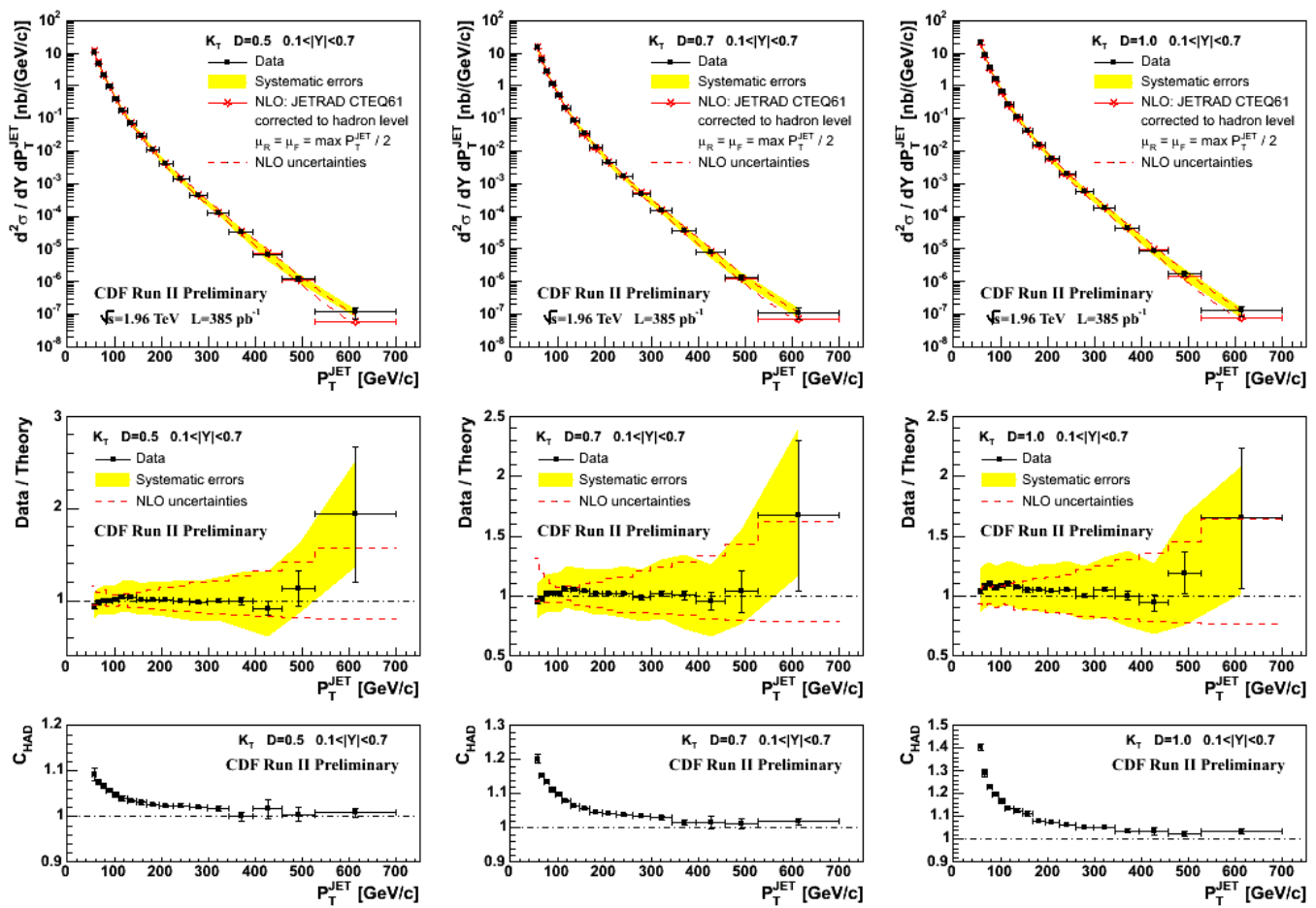

FIGURE 4. The CDF Run 2 inclusive jet cross section using the $\mathrm{K}_{\mathrm{T}}$ algorithm with $\mathrm{D}=0.5,0.7$, and 1.0. The data are at the particle level (with an "underlying event") and the NLO parton level (CTEQ61M) has been corrected for fragmentation effects and for the "underlying event" (with correction factors $\mathrm{C}_{\mathrm{HAD}}$ ).

A third approach for comparing what is measured at the particle level in the detector with the NLO parton level theory is to use the QCD Monte-Carlo models to correct the NLO parton level theory by adding in the effects of fragmentation and the "underlying event". I prefer this approach. It is much better to correct the theory to the hadron level (with an "underlying event") than it is to extrapolate a perfectly good 
experimental observable to something that is not observable (i.e. parton level). Fig. 4 shows the CDF Run 2 inclusive jet cross section using the $\mathrm{K}_{\mathrm{T}}$ algorithm. Here the data are at the particle (i.e. hadron level) and the NLO parton level theory has been corrected to the particle level. As for the MidPoint algorithm, the parton level to hadron level corrections are significant for $\mathrm{P}_{\mathrm{T}}(\mathrm{jet})<300 \mathrm{GeV} / \mathrm{c}$ (coming mostly from the "underlying event"). The agreement between the theory and data is good. Most theorists prefer the $\mathrm{K}_{\mathrm{T}}$ algorithm over cone algorithms, however, it must be demonstrate that the $\mathrm{K}_{\mathrm{T}}$ algorithm will work in the collider environment where there is an "underlying event". Fig. 4 shown that the $\mathrm{K}_{\mathrm{T}}$ algorithm works fine at the Tevatron The parton to hadron correction factors for the $\mathrm{K}_{\mathrm{T}}$ algorithm are similar to the MidPoint algorithm correction factors.
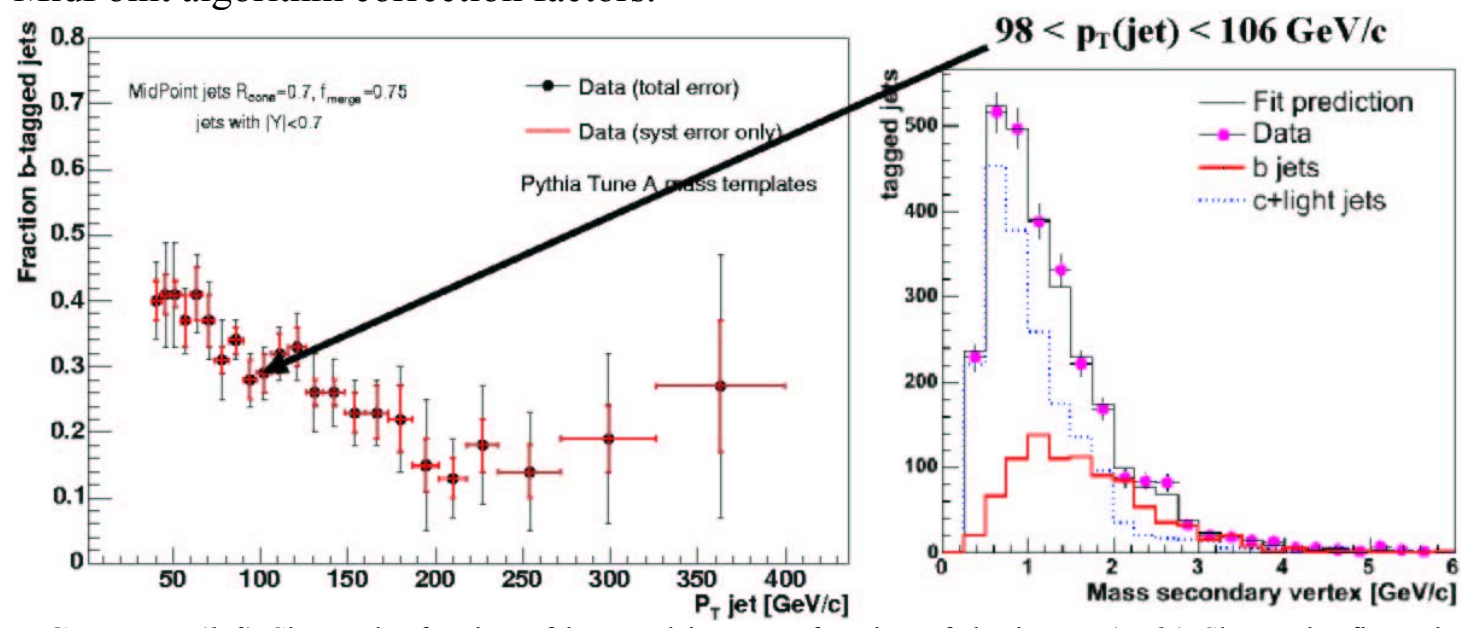

FIGURE 5. (left) Shows the fraction of b-tagged jets as a function of the jet $\mathrm{p}_{\mathrm{T}}$. (right) Shows the fit to the secondary vertex mass for the bin $98<\mathrm{p}_{\mathrm{T}}(\mathrm{jet})<106 \mathrm{GeV} / \mathrm{c}$.
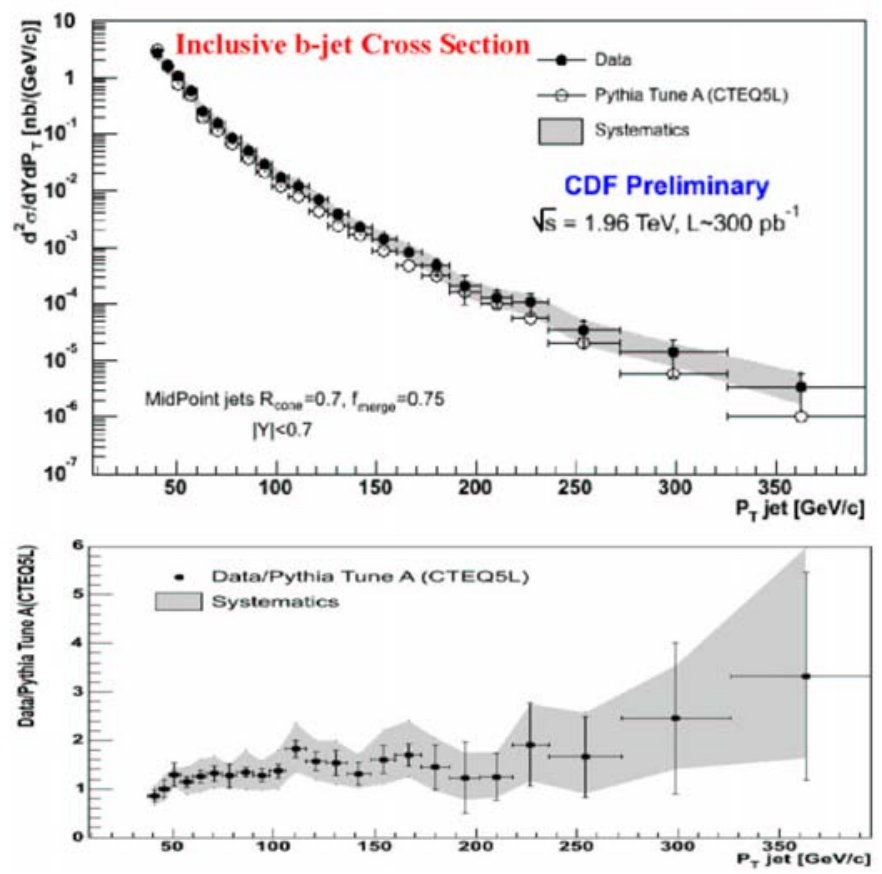

FIGURE 6. (top) Shows the CDF Run 2 b-jet inclusive cross section at $1.96 \mathrm{TeV}$ compared with PYTHIA Tune A. (bottom) Shows the ratio data/theory for PYTHIA Tune A. 
CDF/PHYS/JET/PUBLIC/7898

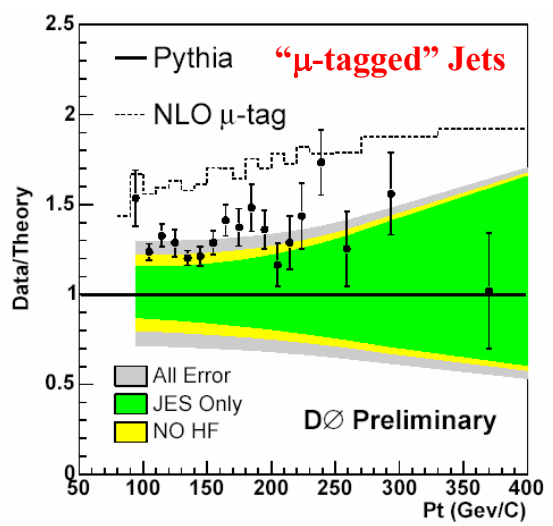

FIGURE 7. Shows the DO Run 2 " $\mu$-tagged" jet inclusive cross section at $1.96 \mathrm{TeV}$ compared with PYTHIA and NLO QCD.

At CDF b-jets are identified by studying the invariant mass of the charged particles emanating from the secondary vertex which is displaced slightly from the primary interaction vertex due to the long lifetime of the heavy b-quark. As shown in Fig. 5, the fraction of b-tagged jets is determined by fitting (on a bin-by-bin bases) the secondary vertex invariant mass to templates determined from PYTHIA Tune A [2-3]. Fig. 6 shows the resulting CDF b-jet inclusive cross section at $1.96 \mathrm{TeV}$ compared with PYTHIA Tune A. The ratio of the data to PYTHIA Tune A is constant with a value of about 1.4. We expect that NLO corrections will account the factor of 1.4

At D0 they study heavy flavor jets by requiring a muon in a jet (i.e. inside $\mathrm{R}=0.5$ ). Searching for muons in jets enhances the heavy flavor content of the jet. Fig 7 shows the DO Run 2 " $\mu$-tagged" jet inclusive cross section compared with PYTHIA Tune A and NLO QCD. The ratio of the data to PYTHIA is constant with a value of about 1.2 , which is similar to the CDF result.
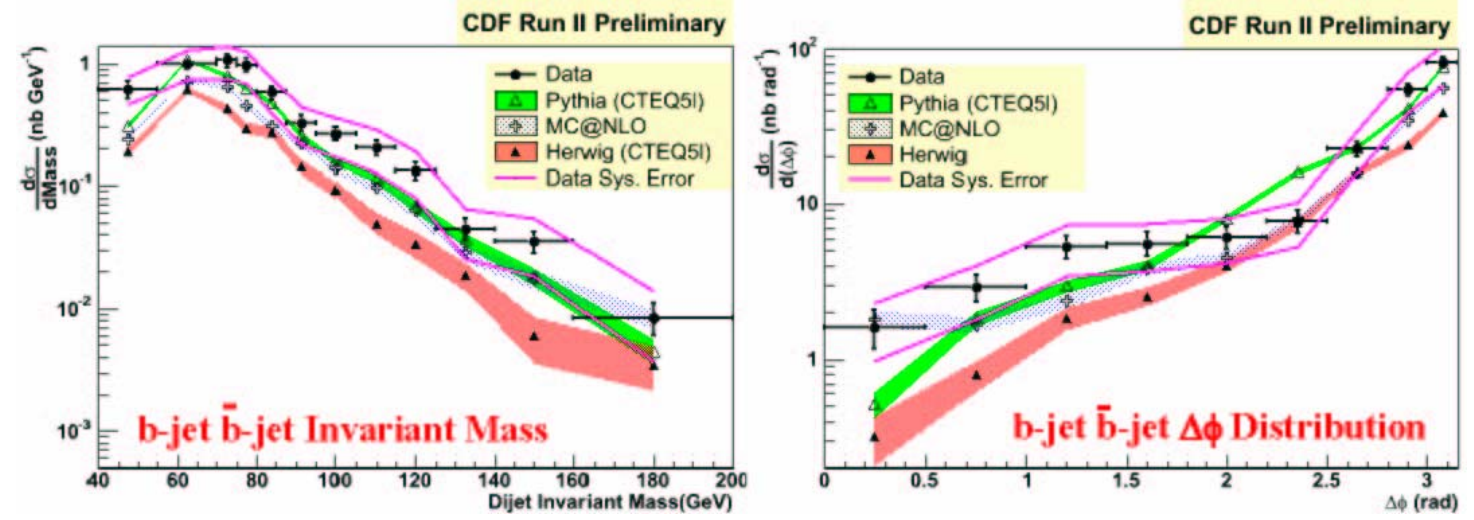

FIGURE 8. Shows the CDF Run $2 b \bar{b}$ dijet invariant mass distribution (left) and the $b$-jet $\bar{b}$-jet $\Delta \phi$ distribution (right) at $1.96 \mathrm{TeV}$ compared with PYTHIA Tune A, HERWIG, and MC@NLO.

Fig. 8 shows the CDF Run $2 b \bar{b}$ dijet invariant mass distribution at $1.96 \mathrm{TeV}$ compared with PYTHIA Tune A, HERWIG [4], and MC@NLO [5] and Table 1 shows the integrated $b \bar{b}$ dijet cross section. PYTHIA Tune A fits the data better than HERWIG or MC@NLO. The is because PYTIA Tune A has been tuned to fit the "underlying event" at the Tevatron by adjusting the multiple-parton interactions. 
HERWIG and MC@NLO (with uses HERWIG) do not include multiple-parton interactions do not have enough activity in the "underlying event". JIMMY [6] is a model of multiple parton interaction which can be combined with HERWIG (and MC@NLO) to enhance the "underlying event" thereby improving the agreement with data. When JIMMY is added to MC@NLO then agreement is improved. Both the inclusive jet cross section and the b-jet cross section depend sensitively on the "underlying event".

Table 1. The CDF Run 2 integrated $b \bar{b}$ dijet cross section $\left(\mathrm{E}_{\mathrm{T}}\left(\mathrm{b}\right.\right.$-jet\#1) $>30 \mathrm{GeV}, \mathrm{E}_{\mathrm{T}}(\mathrm{b}$-jet\#2) $>20 \mathrm{GeV}, \mid \eta$ (b-jets $) \mid<1.2)$ at $1.96 \mathrm{TeV}$ compared with PYTHIA Tune A, HERWIG, MC@NLO, and MC@NLO+ JIMMY).

\begin{tabular}{|r|c|}
\hline CDF (preliminary) & $34.5 \pm 1.8 \pm 10.5 \mathrm{nb}$ \\
\hline PYTHIATuneA (CTEQ5L) & $38.7 \pm 0.6 \mathrm{nb}$ \\
\hline HERWIG (CTEQ5L) & $21.5 \pm 0.7 \mathrm{nb}$ \\
\hline \hline MC@NLO & $28.5 \pm 0.6 \mathrm{nb}$ \\
\hline MC@NLO+ JIMMY & $35.7 \pm 2.0 \mathrm{nb}$ \\
\hline
\end{tabular}

Fig. 8 also shows the $b$-jet $\bar{b}$-jet $\Delta \phi$ distribution compared with PYTHIA Tune A, HERWIG, and MC@NLO. PYTHIA Tune A and MC@NLO do a good job in describing the $b \bar{b} \Delta \phi$ distribution. It is not an accident that PYTHIA Tune A roughly agrees with the data. I tuned the initial-state radiation in PYTHIA Tune A (i.e. PARP(67)) to agree with the CDF Run $1 b \bar{b} \Delta \phi$ distribution [7]. For MC@NLO the agreement is a prediction. For PYTHIA Tune A the agreement is a "tune", but it does show consistency between the CDF Run 1 analysis and the preliminary Run 2 results.

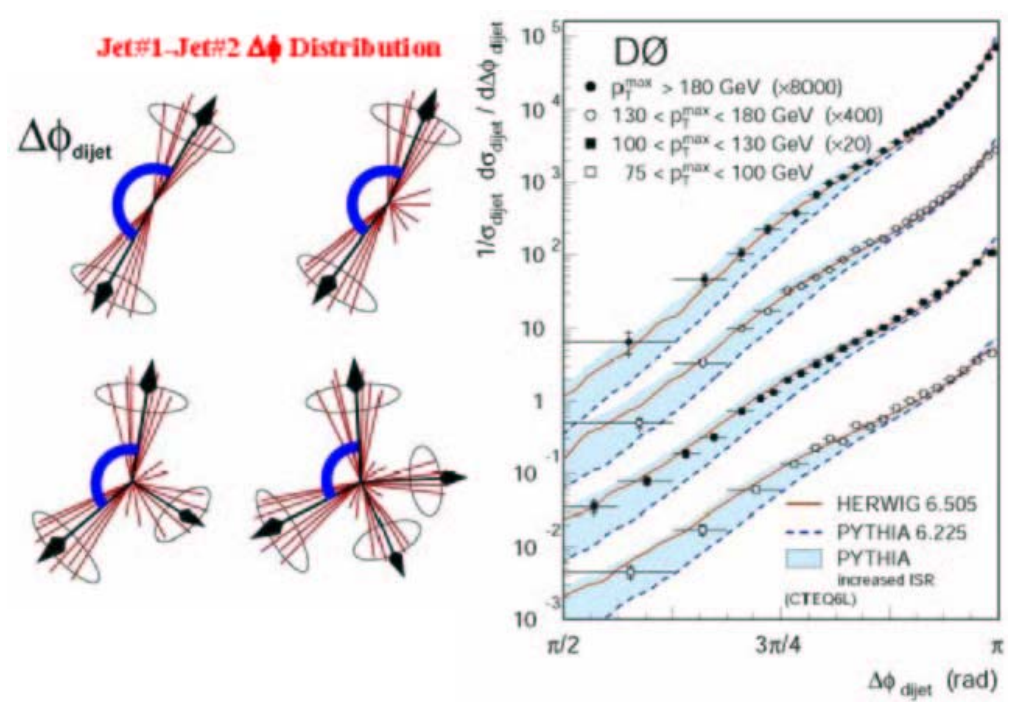

FIGURE 9. Shows the D0 Run 2 jet\#1-jet\#2 $\Delta \phi$ distribution at $1.96 \mathrm{TeV}$ compared with PYTHIA (default) and PYTHIA Tune A (upper edge of the shaded regions). Jet\#1 and jet\#2 are the leading two jets (MidPoint algorithm, $\left.\mathrm{R}=0.7, \mathrm{f}_{\text {merge }}=0.5\right)$. 
Fig. 9 shows the D0 Run 2 jet\#1-jet\#2 $\Delta \phi$ distribution at $1.96 \mathrm{TeV}$, where jet\#1 and jet\#2 are the leading two jets. Here again PYTHIA Tune A does a good job in describing the data.
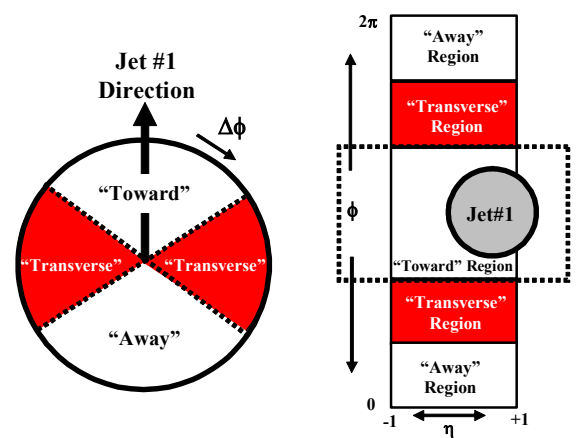

FIGURE 10. Illustration of correlations in azimuthal angle $\Delta \phi$ relative to the direction of the leading jet (MidPoint, $\mathrm{R}=0.7, \mathrm{f}_{\text {merge }}=0.75$ ) in the event, jet $\# 1$. The angle $\Delta \phi=\phi-\phi j e t \# 1$ is the relative azimuthal angle between charged particles (or calorimeter towers) and the direction of jet\#1. The "transverse" region is defined by $60^{\circ}<|\Delta \phi|<120^{\circ}$ and $|\eta|<1$. We examine charged particles in the range $\mathrm{p}_{\mathrm{T}}>0.5 \mathrm{GeV} / \mathrm{c}$ and $|\eta|<1$ and calorimeter towers with $\mathrm{E}_{\mathrm{T}}>0.1 \mathrm{GeV}$ and $|\eta|<1$, but allow the leading jet to be in the region $|\eta(j e t \# 1)|<2$.
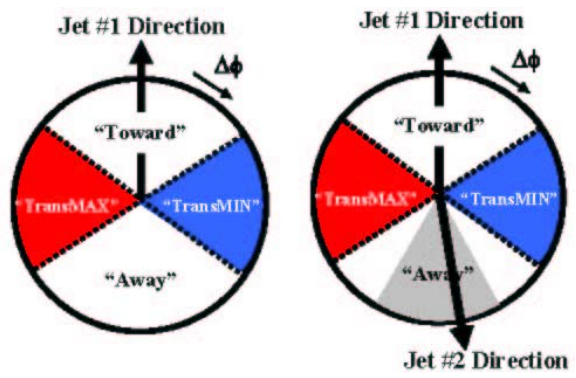

FIGURE 11. Illustration of correlations in azimuthal angle $\Delta \phi$ relative to the direction of the leading jet (highest $\mathrm{P}_{\mathrm{T}}$ jet) in the event, jet\#1 for "leading jet" events (left) and "back-to-back" events (right). Events in which there are no restrictions placed on the on the second highest $\mathrm{P}_{\mathrm{T}}$ jet, jet\#2, are referred to as "leading jet" events. Events with at least two jets where the leading two jets are nearly "back-to-back" $\left(\Delta \phi_{12}>150^{\circ}\right)$ with $\mathrm{P}_{\mathrm{T}}(\mathrm{jet} \# 2) / \mathrm{P}_{\mathrm{T}}(\mathrm{jet} \# 1)>0.8$ and $\mathrm{P}_{\mathrm{T}}($ jet\#3) $<15 \mathrm{GeV} / \mathrm{c}$ are referred to as "back-to-back" events. In both cases the angle $\Delta \phi=\phi-\phi j e t \# 1$ is the relative azimuthal angle between charged particles (or calorimeter towers) and the direction of jet\#1. On an event by event basis, we define "transMAX" ("transMIN") to be the maximum (minimum) of the two "transverse" regions, $60^{\circ}<\Delta \phi<120^{\circ}$ and $60^{\circ}<-\Delta \phi<120^{\circ}$. "TransMAX" and "transMIN" each have an area in $\eta-\phi$ space of $\Delta \eta \Delta \phi=4 \pi / 6$. The overall "transverse" region defined in Fig. 10 includes both the "transMAX" and the "transMIN" region.

We have seen that both the inclusive jet cross section and the b-jet cross section depend sensitively on the "underlying event". At CDF we are working to understand and model the "underlying event" at the Tevatron. We use the topological structure of hadron-hadron collisions to study the "underlying event" [8-10]. The direction of the leading calorimeter jet is used to isolate regions of $\eta-\phi$ space that are sensitive to the "underlying event". As illustrated in Fig. 10, the direction of the leading jet, jet\#1, is used to define correlations in the azimuthal angle, $\Delta \phi$. The angle $\Delta \phi=\phi-\phi_{\text {jet\# } 1 \text { is }}$ the relative azimuthal angle between a charged particle (or a calorimeter tower) and the direction of jet\#1. The "transverse" region is perpendicular to the plane of the hard 2to-2 scattering and is therefore very sensitive to the "underlying event". Furthermore, we consider two classes of events. We refer to events in which there are no 
restrictions placed on the second and third highest $\mathrm{P}_{\mathrm{T}}$ jets (jet\#2 and jet\#3) as "leading jet" events. Events with at least two jets with $\mathrm{P}_{\mathrm{T}}>15 \mathrm{GeV}$ where the leading two jets are nearly "back-to-back" $\left(\left|\Delta \phi_{12}\right|>150^{\circ}\right)$ with $\mathrm{P}_{\mathrm{T}}($ jet $\# 2) / \mathrm{P}_{\mathrm{T}}($ jet $\# 1)>0.8$ and $\mathrm{P}_{\mathrm{T}}($ jet $\# 3)$ $<15 \mathrm{GeV}$ are referred to as "back-to-back" events. "Back-to-back" events are a subset of the "leading jet" events. The idea here is to suppress hard initial and finalstate radiation thus increasing the sensitivity of the "transverse" region to the "beambeam remnant" and the multiple parton scattering component of the "underlying event".

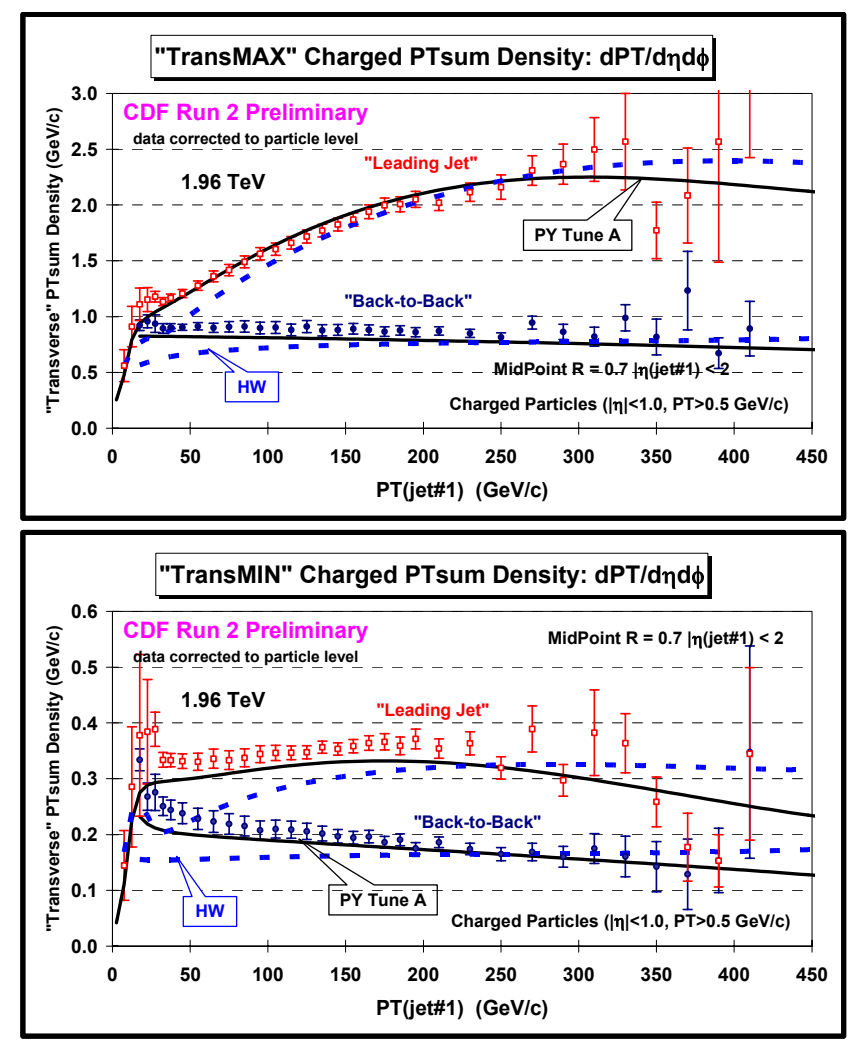

FIGURE 12. CDF Run 2 data at $1.96 \mathrm{TeV}$ on scalar PTsum density of charged particles, dPTsum/d $\eta d \phi$, with $\mathrm{p}_{\mathrm{T}}>$ $0.5 \mathrm{GeV} / \mathrm{c}$ and $|\eta|<1$ in the "transMAX" region (top) and the "transMIN" region (bottom) for "leading jet" and "back-to-back" events defined in Fig. 11 as a function of the leading jet $\mathrm{P}_{\mathrm{T}}$ compared with PYTHIA Tune A and HERWIG. The data are corrected to the particle level (with errors that include both the statistical error and the systematic uncertainty) and compared with the theory at the particle level (i.e. generator level).

As illustrated in Fig. 11, we define a variety of MAX and MIN "transverse" regions which helps separate the "hard component" (initial and final-state radiation) from the "beam-beam remnant" component. MAX (MIN) refer to the "transverse" region containing the largest (smallest) scalar $\mathrm{p}_{\mathrm{T}}$ sum of charged particles or the region containing the largest (smallest) scalar $\mathrm{E}_{\mathrm{T}}$ sum of particles. Since we will be studying regions in $\eta-\phi$ space with different areas, we construct densities by dividing by the

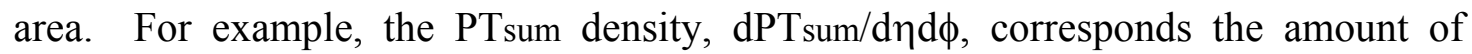
charged particle $\left(\mathrm{p}_{\mathrm{T}}>0.5 \mathrm{GeV} / \mathrm{c}\right)$ scalar $\mathrm{p}_{\mathrm{T}}$ sum per unit $\eta-\phi$, and the transverse energy density, $\mathrm{dE}_{\mathrm{T}} / \mathrm{d} \eta \mathrm{d} \phi$, corresponds the amount of scalar $\mathrm{E}_{\mathrm{T}}$ sum of all particles per unit $\eta$ $\phi$. One expects that "transMAX" will pick up the hardest initial or final-state radiation while both "transMAX" and "transMIN" should receive "beam-beam 
remnant" contributions. Hence one expects "transMIN" to be more sensitive to the "beam-beam remnant" component of the "underlying event", while the "transMAX" minus the "transMIN" (i.e. "transDIF") is very sensitive to initial and final-state radiation. This idea, was first suggested by Bryan Webber, and implemented by in a paper by Jon Pumplin [11]. Also, Valaria Tano studied this in her CDF Run 1 analysis of maximum and minimum transverse cones [12].

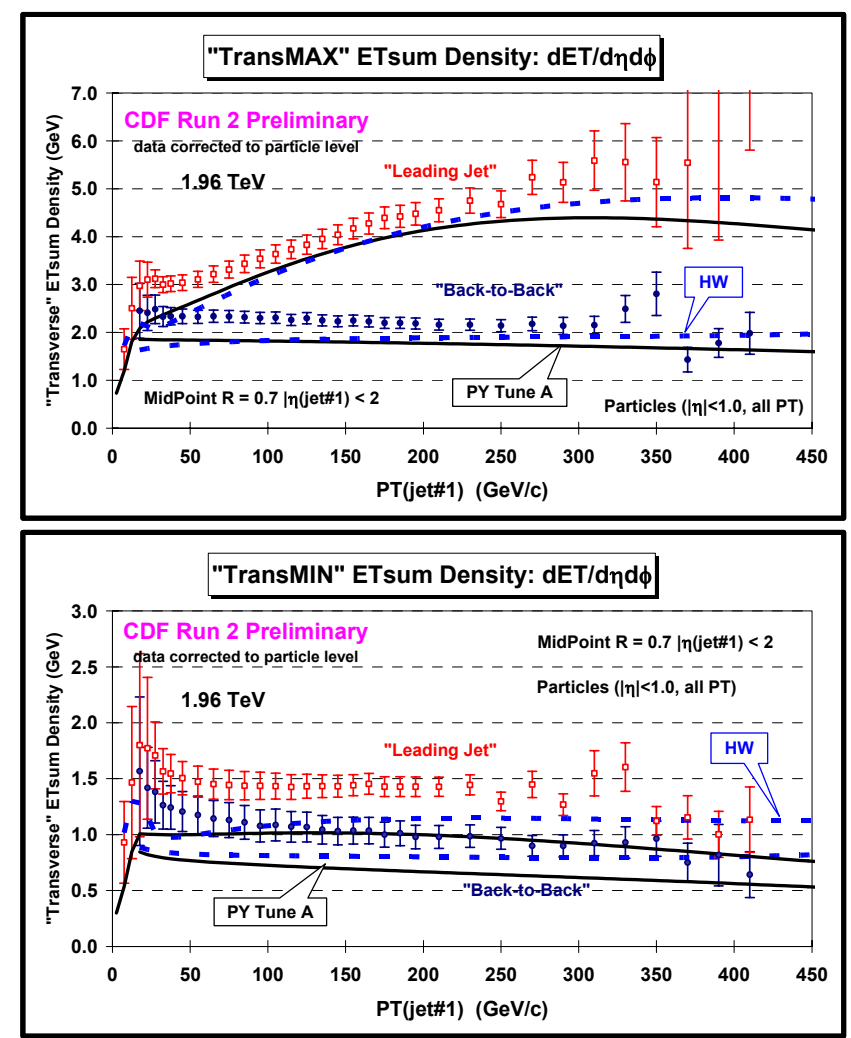

FIGURE 13. CDF Run 2 data at $1.96 \mathrm{TeV}$ on the ETsum density, $\mathrm{dE}_{\mathrm{T}} / \mathrm{d} \eta \mathrm{d} \phi$, for particles with $|\eta|<1$ in the "transMAX" region (top) and the "transMIN" region (bottom) for "leading jet" and "back-to-back" events defined in Fig. 11 as a function of the leading jet $\mathrm{P}_{\mathrm{T}}$ compared with PYTHIA Tune A and HERWIG. The data are corrected to the particle level (with errors that include both the statistical error and the systematic uncertainty) and compared with the theory at the particle level (i.e. generator level).

Fig. 12 compares the data on the density of charged particles and the charged PTsum density in the "transverse" region corrected to the particle level $\left(\mathrm{p}_{\mathrm{T}}>0.5\right)$ for "leading jet" and "back-to-back" events with PYTHIA Tune A and HERWIG at the particle level. As expected, the "leading jet" and "back-to-back" events behave quite differently. For the "leading jet" case the "transMAX" densities rise with increasing $\mathrm{P}_{\mathrm{T}}(\mathrm{jet} \# 1)$, while for the "back-to-back" case they fall with increasing $\mathrm{P}_{\mathrm{T}}(\mathrm{jet} \# 1)$. The rise in the "leading jet" case is, of course, due to hard initial and final-state radiation, which has been suppressed in the "back-to-back" events. The "back-to-back" events allow for a more close look at the "beam-beam remnant" and multiple parton scattering component of the "underlying event" and PYTHIA Tune A (with multiple parton interactions) does a better job describing the data than HERWIG (without multiple parton interactions). 
The "transMIN" densities are more sensitive to the "beam-beam remnant" and multiple parton interaction component of the "underlying event". The "back-to-back" data show a decrease in the "transMIN" densities with increasing $\mathrm{P}_{\mathrm{T}}(\mathrm{jet} \# 1)$ which is described fairly well by PYTHIA Tune A (with multiple parton interactions) but not by HERWIG (without multiple parton interactions). The decrease of the "transMIN" densities with increasing $\mathrm{P}_{\mathrm{T}}(\mathrm{jet} \# 1)$ for the "back-to-back" events is very interesting and might be due to a "saturation" of the multiple parton interactions at small impact parameter. Such an effect is included in PYTHIA Tune A but not in HERWIG (without multiple parton interactions).
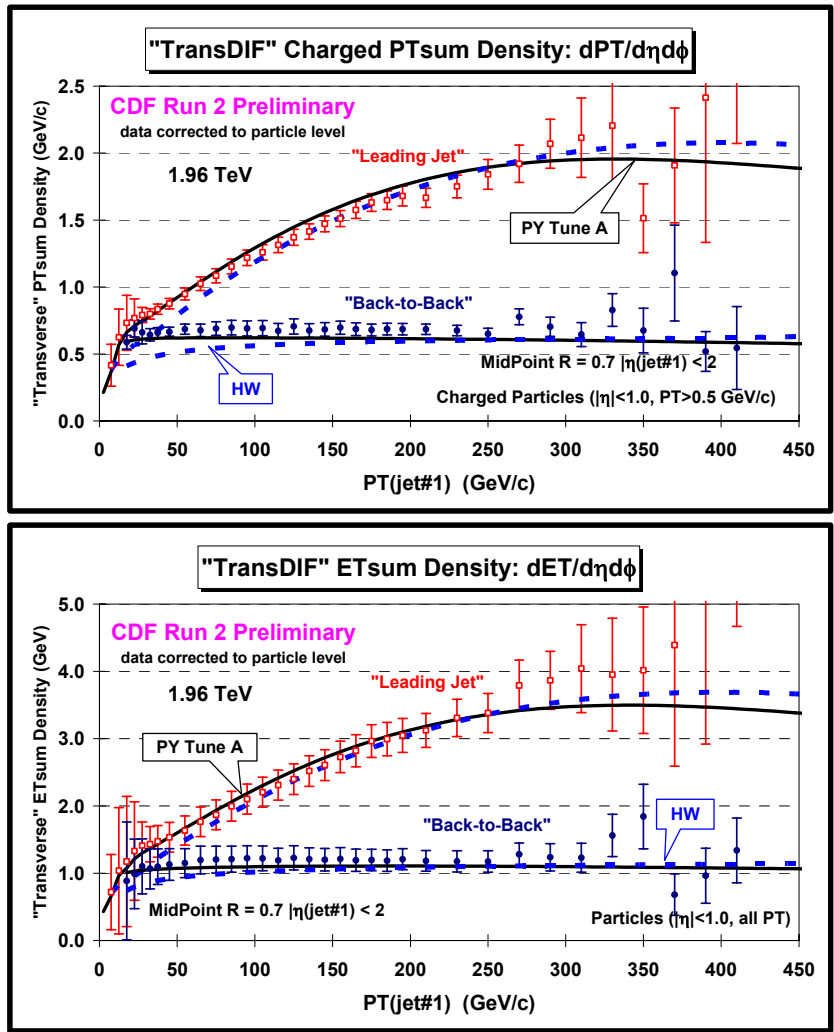

FIGURE 14. CDF Run 2 data at $1.96 \mathrm{TeV}$ on the difference of the "transMAX" and "transMIN" region ("transDIF" = "transMAX" minus "transMIN") for "leading jet" and "back-to-back" events defined in Fig. 11 as a function of the leading jet $\mathrm{P}_{\mathrm{T}}$ compared with PYTHIA Tune A and HERWIG. The data are corrected to the particle level (with errors that include both the statistical error and the systematic uncertainty) and compared with the theory at the particle level (i.e. generator level).

Fig. 13 shows the data corrected to the particle level for the ETsum density, $\mathrm{dE}_{\mathrm{T}} / \mathrm{d} \eta \mathrm{d} \phi$, in the "transverse" region for "leading jet" and "back-to-back" events compared with PYTHIA Tune A and HERWIG at the particle level. The data on scalar ETsum density has been corrected to correspond to all particles (all $\mathrm{p}_{\mathrm{T}},|\eta|<1$ ). Neither PYTHIA Tune A or HERWIG produce enough energy in the "transverse" region. HERWIG has more "soft" particles than PYTHIA Tune A does slightly better in describing the energy density in the "transMAX" and "transMIN" region.

Fig. 14 shows the difference of the "transMAX" and "transMIN" region ("transDIF" = "transMAX" minus "transMIN") for "leading jet" and "back-to-back" events compared with PYTHIA Tune A and HERWIG. "TransDIF" is more sensitive 
to the hard scattering component of the "underlying event" (i.e. initial and final state radiation).

\section{CONCLUSIONS}

We have measured the inclusive jet cross section at the Tevatron using the MidPoint algorithm with $\mathrm{R}=0.7$ and $\mathrm{f}_{\text {merge }}=0.5$ (D0), using the MidPoint algorithm with $\mathrm{R}=0.7$ and $\mathrm{f}_{\text {merge }}=0.75(\mathrm{CDF})$, and using the $\mathrm{K}_{\mathrm{T}}$ algorithm(CDF). The data agree well with the NLO parton-level theory after the data is extrapolated to the parton-level or the parton-level theory is corrected for the "underlying event" and fragmentation. The $\mathrm{K}_{\mathrm{T}}$ algorithm works fine at the Tevatron collider, which has positive implications for the LHC.

We have also measured the b-jet inclusive cross section and the $b \bar{b}$ dijet invariant mass distribution. There measurements at hadron colliders provide an important test of QCD. Past Tevatron measurements of b-quark production indicated a possible "excess" with respect to QCD predictions. However, the b-jet cross is in agreement expectations. The data are about a factor of 1.4 larger than the prediction of PYTHIA Tune A, however, this is to be expected since PYTHIA is a "leading log order" model. One cannot expect it correctly predict the precise amount of "flavor excitation" and "gluon splitting". We are working on the comparisons with MC@NLO [5].

CDF has done extensive studies of the "underlying event" at the Tevatron. PYTHIA Tune A (with multiple parton interactions) does a good job in describing the charged particles $\left(\mathrm{p}_{\mathrm{T}}>0.5 \mathrm{GeV} / \mathrm{c},|\eta|<1\right)$ in the "underlying event" (i.e. "transverse" regions) for both "leading jet" and "back-to-back" events. HERWIG (without multiple parton interactions) does not have enough activity in the "underlying event" for $\mathrm{P}_{\mathrm{T}}(\mathrm{jet} \# 1)$ less than about $150 \mathrm{GeV}$. Both PYTHIA Tune A and HERWIG underestimate the energy density in the "transMAX" and "transMIN" regions. However, they both fit the "transDIF" energy density. This indicates that the excess energy density seen in the data probably arises from the "soft" component of the "underlying event" (i.e. beam-beam remnants and/or multiple parton interactions).

We see interesting dependence of the "underlying event" on the transverse momentum of the leading jet (i.e. the $\mathrm{Q}^{2}$ of the hard scattering). For the "leading jet" case the "transMAX" densities rise with increasing $\mathrm{P}_{\mathrm{T}}(\mathrm{jet} \# 1)$, while for the "back-toback" case they fall with increasing $\mathrm{P}_{\mathrm{T}}(\mathrm{jet} \# 1)$. The rise in the "leading jet" case is due to hard initial and final-state radiation, which has been suppressed in the "back-toback" events. The "back-to-back" data show a decrease in the "transMIN" densities with increasing $\mathrm{P}_{\mathrm{T}}(\mathrm{jet} \# 1)$. The decrease of the "transMIN" densities with increasing $\mathrm{P}_{\mathrm{T}}(\mathrm{jet} \# 1)$ for the "back-to-back" events is very interesting and might be due to a "saturation" of the multiple parton interactions at small impact parameter. Such an effect is included in PYTHIA Tune A (with multiple parton interactions) but not in HERWIG (without multiple parton interactions). PYTHIA Tune A does predict this decrease, while HERWIG shows an increase (due to increasing initial and final state radiation). 
CDF/PHYS/JET/PUBLIC/7898

\section{REFERENCES}

1. S. D. Ellis and D. E. Soper, Phys. Rev. D48, 3160 (1993).

2. Min-Bias and the Underlying Event at the Tevatron and the LHC, talk by R. Field at the Fermilab ME/MC Tuning Workshop, Fermilab, October 4, 2002. Toward an Understanding of Hadron Collisions: From Feynman-Field until Now, talk by R. Field at the Fermilab Joint Theoretical Experimental "Wine \& Cheese" Seminar, Fermilab, October 4, 2002.

3. T. Sjostrand, Phys. Lett. 157B, 321 (1985); M. Bengtsson, T. Sjostrand, and M. van Zijl, Z. Phys. C32, 67 (1986); T. Sjostrand and M. van Zijl, Phys. Rev. D36, 2019 (1987).

4. G. Marchesini and B. R. Webber, Nucl. Phys B310, 461 (1988); I. G. Knowles, Nucl. Phys. B310, 571 (1988); S. Catani, G. Marchesini, and B. R. Webber, Nucl. Phys. B349, 635 (1991).

5. The MC and NLO 3.1 Event Generator, Stefano Frixione and Bryan R. Webber, CAVENDISH-HEP-05-09, hep-ph/0506182 (2005). Matching NLO QCD and Parton Showers in Heavy Flavor Production, Stefano Frixione, Paolo Nason, and Bryan R. Webber, JHEP 0308:007 (2003).

6. Multiparton Interactions in Photoproduction at HERA, J.M. Butterworth, J.R. Forshaw, and M.H. Seymour, Z. Phys. C7, 637-646 (1996).

7. Measurements of Bottom Anti-Botootm Azimuthal Production Correlations in Proton-Antiproton Collisions at 1.8 TeV, CDF Collaboration (D. Acosta et al.), Phys. Rev. D71, 092001 (2005),

8. Charged Jet Evolution and the Underlying Event in Proton-Antiproton Collisions at 1.8 TeV, The CDF Collaboration (T. Affolder et al.), Phys. Rev. D65, 092002 (2002).

9. The Underlying Event in Large Transverse Momentum Charged Jet and Z-boson Production at 1.8 TeV, talk presented by Rick Field at DPF2000, Columbus, OH, August 11, 2000.

10. A Comparison of the Underlying Event in Jet and Min-Bias Events, talk presented by Joey Huston at DPF2000, Columbus, $\mathrm{OH}$, August 11, 2000. The Underlying Event in Jet and Minimum Bias Events at the Tevatron, talk presented by Valeria Tano at ISMD2001, Datong, China, September 1-7, 2001.

11. Hard Underlying Event Corrections to Inclusive Jet Cross-Sections, Jon Pumplin, Phys. Rev. D57, 5787 (1998).

12. The Underlying Event in Hard Interactions at the Tevatron Proton-Antiproton Collider, CDF Collaboration (D. Acosta et al.), Phys. Rev. D70, 072002 (2004). 